\title{
Unintended allocation of spatial attention to goal-relevant but not to goal-related events
}

Article

Accepted Version

Vogt, J., De Houwer, J. and Moors, A. (2011) Unintended allocation of spatial attention to goal-relevant but not to goalrelated events. Social Psychology, 42 (1). pp. 48-55. ISSN 1864-9335 doi: https://doi.org/10.1027/1864-9335/a000042 Available at https://centaur.reading.ac.uk/68433/

It is advisable to refer to the publisher's version if you intend to cite from the work. See Guidance on citing.

To link to this article DOI: http://dx.doi.org/10.1027/1864-9335/a000042

Publisher: Hogrefe

All outputs in CentAUR are protected by Intellectual Property Rights law, including copyright law. Copyright and IPR is retained by the creators or other copyright holders. Terms and conditions for use of this material are defined in the End User Agreement.

www.reading.ac.uk/centaur 
Central Archive at the University of Reading

Reading's research outputs online 
Running head: Unintended attending to goal-relevant but not to goal-related events

Unintended allocation of spatial attention to goal-relevant but not to goal-related events

Julia Vogt, Jan De Houwer, and Agnes Moors

Department of Experimental-Clinical and Health Psychology, Ghent University, Belgium

Accepted for publication: Social Psychology [Special Issue on the Limits of Intentionality].

WORD COUNT (main text, footnotes): 4987

Correspondence Address:

Julia Vogt

Department of Experimental-Clinical and Health Psychology

Ghent University

Henri Dunantlaan 2

B-9000 Ghent (Belgium)

E-mail: julia.vogt@UGent.be

Tel: ++32-9-264.94.43 Fax: ++32-9-264.64.89 


\begin{abstract}
We investigated whether words relevant to a person's current goal and words related to this goal influence the orienting of attention even when an intention to attend to the goal-relevant and goal-related stimuli is not present. Participants performed a modified spatial cueing paradigm combined with a second task that induced a goal. The results showed that the induced goal led to the orienting of attention to goal-relevant words in the spatial cueing task. This effect was not found for goal-related words. The results provide evidence for accounts of automatic goal pursuit, which state that goals automatically guide attention to goal-relevant events.
\end{abstract}

Keywords: spatial attention; attentional bias; goals; motivation; intentions 
The last two decades have been marked by an enormous amount of research showing that goals often are activated and pursued automatically (Bargh, 1997; Shah, 2005). Large parts of this research focused on the unconscious activation of goals (e.g., Chartrand \& Bargh, 1996; Bargh, Gollwitzer, Lee-Chai, Barndollar, \& Troetschel, 2001). But even when goals are activated consciously, the pursuit of a goal will be accompanied by the operation of automatic processes (for an overview see Ferguson, Hassin, \& Bargh, 2008). For instance, it has been shown that goal pursuit goes together with the automatic activation of goal-relevant information (Förster, Friedman, \& Higgins, 2005) and the automatic inhibition of distracting information such as information relevant to conflicting goals (Shah, Friedman, \& Kruglanski, 2002). Moreover, Moors and De Houwer (2001) found that goal-congruent events evoke spontaneous positive evaluations whereas goal-incongruent events lead to spontaneous negative evaluations.

In a stimulus-overloaded world, it is of particular importance that goals point the individual at goal-relevant events in the environment. Attention is regarded as the mechanism that allows focusing on a subset of possible sensory inputs or trains of thought (Luck \& Vecera, 2002). Hence, attention is the process that filters which inputs will be further processed and which will not. Prominent accounts of automatic goal pursuit therefore assume that goalrelevant events attract attention automatically (Moskowitz, Li, \& Kirk, 2004; Ferguson et al., 2008). In the present study, we investigated this hypothesis concerning two features of automaticity (for a discussion of automaticity features see Bargh, 1994, or Moors \& De Houwer, 2006), namely whether goal-relevant events will be attended to fast and unintentionally, that is, without an intention to attend to them.

\section{Goal-Driven but Unintended Attending}

The idea that attention is for a large part goal-driven (i.e., allocated to goal-relevant information) can be found not only in models of goal pursuit but also in the literature on 
attention (e.g., Yantis, 1998; Corbetta \& Shulman, 2002). In this field, goal-driven attention is often defined as the controlling of attention by the observer's deliberate strategies and intentions (Yantis, 1998). However, this definition leaves open whether the goal that drives attention is (a) a goal about the process of attending (e.g., directing attention towards a stimulus) or (b) a goal that does not imply the attentional process but that could influence it. Illustrating the first option, the goal of wanting to eat an apple could cause a second goal, namely an intention to direct attention towards apples in the fruit basket. Note that we define intention as the goal to engage in a process (Moors \& De Houwer, 2006), in this case, as the goal to engage in the process of attending to goal-relevant events (i.e., apples in the fruit basket). In this example, attending to goal-relevant events would be intended and in this respect not automatic. In contrast, we hypothesize that goal-relevant events will also be attended to even when attending is not caused by an intention to attend to goal-relevant events. For instance, an apple in another location in the room might grab one's attention although one only intended to attend to apples in the fruit basket. In this case, attention is driven by a goal (i.e., to eat an apple) without that this goal implied attending to this particular stimulus.

\section{Goal-Related Events}

A second issue we are interested in is the kind of events that are attended to when goals guide the unintended allocation of attention. Consider the goal of wanting to eat an apple. In this case, apples are goal-relevant events. We would therefore predict that apples are attended to automatically. In addition to truly goal-relevant events, there is also a wide range of events that are merely related to the goal of eating apples, such as other fruits, apple juice, or apple pie. There might be situations in which attending to both goal-relevant and goal-related events is useful. For instance, when one is hungry and therefore has the goal of eating an apple, apple pie and other fruits can fulfill the same need. However, it would probably be dysfunctional when 
attention would be allocated unconditionally to any goal-related event, especially when the goal is specific and goal-relevant events are therefore well defined (e.g., the individual only wants to eat an apple).

Based on a similar reasoning, Veling and van Knippenberg (2006, see also 2008) investigated whether only goal-relevant or also goal-related knowledge is activated during the pursuit of a specific goal. They conducted an experiment in which participants pursued the goal of reacting to a specific subset of exemplars (e.g., apples and bananas) from one semantic category (e.g., fruits). These authors found that representations of other fruit exemplars (e.g., peaches and mangos) were inhibited. Inhibition of goal-related but goal-irrelevant knowledge is assumed to take place because it shields goal pursuit against interference by this knowledge. However, this study does not allow conclusions about whether goal-related events are attended to. It could be that especially early and automatic attentional processes are guided by a mechanism that does not allow a fine-grained distinction between goal-relevant and goal-related events (cf. Duncan \& Humphreys, 1989; Moores, Laita, \& Chelazzi, 2003). In the present study, we therefore investigated whether the fast and unintended allocation of attention to goal-relevant events is accompanied by goal shielding effects for goal-related events.

Previous Evidence Regarding the Unintended Allocation of Attention to Goal-Relevant and Goal-Related Stimuli

A recent study from our lab (Vogt, De Houwer, Moors, Van Damme, \& Crombez, 2010; see also Folk, Remington, \& Johnston, 1992; Vogt, De Houwer, \& Crombez, in press) provided a first test of the hypothesis that goal-relevant but not goal-related events are attended to even in the absence of an intention to attend to them (even when both, goal-relevant and goal-related events, were linked to a motor response). In this study, we combined an attention task with a separate, goal-inducing task. In each trial of the goal task, a single word appeared in the middle 
of the screen. A goal was induced by asking participants to respond (by pressing the spacebar) when one of two particular words (e.g., "field" or "ship") was presented in the middle of the screen. Participants were financially rewarded when they correctly indicated the presence of the two words. Consequently, we regarded these words as goal relevant.

The attention task was a modified spatial cueing paradigm which allows studying covert attentional orienting to peripheral cues (cf. Fox, Russo, Bowles, \& Dutton, 2001; Koster, Crombez, Van Damme, Verschuere, \& De Houwer, 2004). In this task, participants have to detect visual probes presented at the left or right side of a fixation cross. The probe is preceded by a visual cue at the same location (validly cued trials) or opposite location (invalidly cued trials). Probes are usually detected faster on valid than on invalid trials. This effect is typically referred to as the cue validity effect. This effect is more pronounced for salient cues such as emotional stimuli (Fox et al., 2001; Koster et al., 2004). Moreover, this task allows examining whether a cue affects the engagement component of attention (response time benefits for one cue category on valid trials due to the engagement of attention at the validly cued location) or the disengagement component (response time costs due to delayed disengagement of attention from the invalidly cued location), or both (see Cisler \& Koster, 2010).

In order to investigate the unintended allocation of attention to goal-relevant events, we used the goal-relevant words as cues in the spatial cueing paradigm. We also presented goalrelated and control words as cues. Goal-related words were synonyms of the goal-relevant words (e.g., "boat" when "ship" was the goal-relevant word). Trials of the goal task and trials of the cueing task alternated. This procedure allowed measuring the attentional processing of the goal-relevant and goal-related words while participants were simultaneously pursuing the goal. However, because goal and cueing task were two independent tasks, attending to the goalrelevant stimulus in the cueing task was neither necessary nor instrumental for achieving the goal 
implemented in the goal task. Therefore, participants were not encouraged to set up an intention to attend to goal-relevant stimuli in the cueing task. The results of our study showed that attention nevertheless was more strongly oriented to goal-relevant words compared to accessible control words. In contrast, attending to goal-related words did not differ from attending to the control words, suggesting that goal-related words did not attract attention.

\section{The Present Study}

The preliminary study by Vogt et al. (2010) left open two questions that we aimed to address in the present study. First, in the study by Vogt et al., a cue validity ratio of $75 \%$ valid cues was implemented, that is, $75 \%$ of the cues in the cueing task were predictive of the probe location. This aspect of the design cannot be responsible for the fact that attention was more strongly oriented to goal-relevant cues than to other cues because the cue validity ratio made it instrumental to attend to all cue categories. Nevertheless, it is possible that heightened attention for goal-relevant cues occurs only when directing attention to cues in general is instrumental. Hence, in the present study, we examined whether unintended attending to goal-relevant cues occurs also when attending to (goal-relevant or other) cues is not instrumental. We did this by using a cue validity ratio of $50 \%$, so that cues did not predict the probe location above chance level.

Second, we were interested to see whether the effects for goal-related events obtained in the Vogt et al. study were caused by the specific characteristics of the goal in that study. The goal task in that study required participants to respond to only two words that were visually distinct from the other stimuli. Because of this, attentional orienting might have been guided by the visual representation of the goal-relevant words (e.g., the form of the letters of the words). This would explain why semantically similar but visually different words did not attract attention. It is possible, however, that inattention to goal-related stimuli is limited to situations in 
which the semantic meaning of goal-relevant events can be "turned off". In the present study, we used a goal task that forced participants to keep in mind the semantic meaning of the goal stimuli and we investigated attending to goal-related words under these conditions. To this end, participants were asked not only to respond to goal-relevant words (e.g., "ship") but also to pictures that represented the content of the goal-relevant words (i.e., different pictures showing a ship) in the goal task.

\section{Method}

\section{Participants}

Sixteen students (12 women) from different faculties at Ghent University participated. They were paid $5 €$ each. All participants had normal or corrected-to-normal vision and were naive as to the purpose of the experiment.

\section{Apparatus and Materials}

The experiment was programmed and presented using the INQUISIT Millisecond software package (Inquisit 2.0, 2005) on a Dell Dimension 5000 computer with an 85 Hz, 17inch CRT monitor (Resolution: 1024 x 768). All stimuli were presented against a black background.

\section{Stimuli in the cueing task.}

We used the same four word pairs as Vogt et al. (2010): (1) stripe (streep) and work (werk), (2) line (lijn) and labor (arbeid), (3) field (akker) and boat (boot), and (4) land (veld) and ship (schip). Each of the four pairs served one of the following functions in the modified spatial cueing task: (1) goal-relevant cues, (2) goal-related cues, (3) control cues, and (4) control-related cues. The assignment of a function to a word pair was counterbalanced over participants. We used control cues because, in principle, it is possible that attention is oriented to goal-relevant words not because they are goal relevant but because they were mentioned explicitly in the 
instructions. To control for such pre-exposure effects, control words were mentioned and had to be memorized during the instructions. The synonyms of these control words (i.e., control-related cues) were used in the modified spatial cueing paradigm in order to have an appropriate control condition for the synonyms of the goal words (i.e., goal-related cues).

The word pairs were constructed in the following manner. Neutral words were chosen from the database of Hermans and De Houwer (1994), and were completed with neutral words that matched these words concerning word length and frequency as indicated by the WordGen tool (Duyck, Desmet, Verbeke, \& Brysbaert, 2004). The chosen synonyms were checked via the Thesaurus synonym tool of Microsoft Office Word 2003 and with native speakers.

\section{Stimuli in the goal task.}

In the goal task, four categories of stimuli were shown: (1) goal-relevant words, (2) goalrelevant pictures, (3) control words, (4) control pictures, and filler stimuli. Control words and pictures required no reaction in this task but were presented here to make sure that goal-relevant and control stimuli were presented equally often during the experiment. Goal-relevant and control pictures were searched via the internet. For each synonym pair (e.g., work and labor), six pictures were located by using the Dutch words as search term. Additionally, four filler words and 24 filler pictures were used. We used filler stimuli in order to be able to present the stimuli of the goal categories less often so that participants could not learn the visual features of these stimuli. Filler pictures consisted of 12 colored rectangles and 12 pictures from the International Affective Picture System (IAPS; Lang, Bradley, \& Cuthbert, 1999). According to the normative IAPS ratings, these picture were neutral to slightly positive $(M=6.45 ; S D=1.73){ }^{1}$

\section{Modified spatial cueing task.}

Each trial of the modified spatial cueing task started with the presentation of a black fixation cross $(5 \mathrm{~mm}$ high) placed in the middle of a white rectangle $(4.5 \mathrm{~cm}$ high x $5.5 \mathrm{~cm}$ wide) 
in the middle of the screen (Figure 1). Along with this, two other white rectangles of the same size were presented, one to the left and one to the right of the middle rectangle. The middle of each of these two peripheral rectangles was $9 \mathrm{~cm}$ from the fixation cross. Cues and probes were presented within the peripheral rectangles. After $500 \mathrm{~ms}$, a cue word appeared for the duration of $250 \mathrm{~ms}$. Cue words were presented in Arial font size 20. Immediately after cue offset, a probe consisting of a black square $(0.8 \mathrm{~cm}$ x $0.8 \mathrm{~cm})$ was presented. Responses were made by pressing one of two keys (probe left: “ $q$ "; probe right: " 5 ") with the left and right index finger on an AZERTY keyboard. A trial of this task ended after a response was registered or $1500 \mathrm{~ms}$ had elapsed since the onset of the probe. In case an incorrect response was given, the word "ERROR" appeared for $200 \mathrm{~ms}$ in the middle of the screen.

Each cue word and the probe were presented equally often in both spatial locations. To control for responses to cues instead of probes, catch trials were presented. On these trials, a probe did not follow the cue and no response was required. In order to ensure that participants maintained fixation at the middle of the screen, digit trials were presented. On these trials, the fixation cross was followed only by a randomly selected digit between 1 and 9 for a duration of 50 ms. Participants were instructed to report the digit aloud. Responses on digit trials were not registered.

\section{Goal task.}

The goal task started with the appearance of a word (presented in black and Arial font size 20) on a white background in the middle of the screen for $250 \mathrm{~ms}$ (Figure 1). Hereafter, a red question mark ( $8 \mathrm{~mm}$ high) appeared in the middle of the screen, which indicated that participants should press the spacebar when the preceding stimulus in the middle of the screen had been a goal-relevant word or picture. A trial of this task ended with a response or when 2000 ms had elapsed since the onset of the question mark. If a correct response was given after a 
goal-relevant stimulus, the word "CORRECT" appeared for $200 \mathrm{~ms}$ in the middle of the screen. In case an incorrect response was given, the word "ERROR" appeared for $200 \mathrm{~ms}$ in the middle of the screen.

\section{Procedure}

\section{Instructions and practice phase.}

Participants were informed that a computer task would be administered, and gave a written informed consent. They were seated approximately $60 \mathrm{~cm}$ from a computer screen. All further instructions were presented on the computer screen.

For the cueing task, participants were asked to respond as quickly and as accurately as possible to the location of the probe by pressing the corresponding key. They were informed that a cue would precede the presentation of the probe. Participants were instructed to maintain attention at the fixation cross.

Participants were informed that after they responded to the probe of the cueing task, a word would be presented in the middle of the screen. For the practice phase of the goal task, participants were asked to press the spacebar with both thumbs if one particular word had been presented. Participants were informed that no fast reaction was required. In the practice phase, stimuli (five words) different from those used in the test phase were used in the goal and cueing task. The practice phase included 28 trials.

\section{Test phase.}

Before the test phase, participants were presented with four words which were actually the two goal-relevant (e.g., land and ship) and the two control words (e.g., stripe and work) for the test phase. Participants were not informed at this point that these words would be used in the test phase. Participants were asked to memorize the words and to write them down after the words disappeared from the screen. The experimenter checked whether the correct words were 
written down. This was repeated until all words were memorized correctly. Hereafter, participants were told that the test phase would start. The four words were shown again with the information that they would be used in the test phase. Participants were instructed to indicate the presence of two of these words (e.g., land and ship) in the goal task. Moreover, they were told that they were also required to indicate the presence of photos referring to the goal-relevant words (e.g., they were told to react when a photo referring to land or ship would be presented). Participants were informed that they would receive 5 points for correctly indicating the presence of the goal-relevant words and photos in this task.

The test phase consisted of 140 trials of the spatial cueing task and 140 trials of the goal task. For the spatial cueing task these were 128 test trials (32 trials for each of the four cue categories: goal-relevant, goal-related, control, and control-related), eight catch trials, and four digit trials. The 140 trials of the goal task included 16 trials for each category (goal-relevant words, goal-relevant pictures, control words, and control pictures) and 76 filler trials (40 trials with a filler picture, 36 trials with a filler word).

Trials of the modified spatial cueing task and the goal task alternated. The inter-trial interval was $600 \mathrm{~ms}$. The order of trials was determined randomly for both tasks and for each participant separately. The orders of the trials of the cueing task and of the trials of the goal task were selected independently of each other. Hence, the cue words that were presented in a trial of the cueing task were not predictive of the word or picture that appeared in the following trial of the goal task. Moreover, it was randomly determined which picture of the goal-relevant or control picture category was presented in a trial of the goal task, but the task was programmed in such a way that each picture had to be shown once before a picture was shown a second time.

\section{Results}

Trials with errors were removed from the data $(0.63 \%)$. In line with the study of Vogt et 
al. (2010), reaction times (RTs) shorter than $150 \mathrm{~ms}$ and longer than $750 \mathrm{~ms}$ were considered as outliers and excluded from the analysis $(2.68 \%)^{2}$. Means and standard deviations of the responses can be found in Table 1. During the test phase, participants never responded on the catch trials, suggesting that none of the cues was associated with a systematic response bias. Participants made errors on $3.13 \%$ of the trials in the goal task $(24 \%$ of these errors were missed reactions to goal-relevant stimuli, $76 \%$ were false alarms).

We performed a 4 (cue category: goal relevant, goal related, control, control related) x 2 (cue validity: valid, invalid) repeated measures analysis of variance (ANOVA) on the RTs. This analysis revealed a significant effect of cue validity, $F(1,15)=5.07, p<.05, \mathrm{y}^{2}=.252$, with faster reactions on validly cued trials $(M=419 \mathrm{~ms} ; S D=60 \mathrm{~ms})$ compared to invalidly cued trials $(M=432 \mathrm{~ms} ; S D=57 \mathrm{~ms})$. The main effect of category did not reach significance, $F<$ 1.79.

Most importantly, the predicted interaction of cue validity and cue category was significant, $F(3,13)=4.13, p<.03, \mathrm{y}^{2}=.488$. To further explore the interaction, we calculated cue validity indices for each cue category by subtracting RTs on valid trials from RTs on invalid trials (Table 1). Planned comparisons were conducted in order to compare the cue validity indices of the different cue categories. Supporting our hypotheses, the cue validity index for goal-relevant cues was significantly larger than the cue validity index for control cues, $t(15)=$ $2.99, p<.01, d=1.10$. Further, the cue validity index for goal-relevant cues was also significantly larger than the cue validity index for goal-related cues, $t(15)=3.72, p<.01, d=$ 1.33 , and tended to be larger than the cue validity index for control-related cues, $t(15)=1.99, p=$ $.065, d=0.73$. The cue validity indices for control, goal-related, and control-related cues did not differ significantly from each other, $t \mathrm{~s}<1.63, n s$.

In order to investigate whether larger cue validity indices to goal-relevant cues were due 
to faster engagement of attention to goal-relevant cues or to delayed disengagement of attention from goal-relevant cues, or to both processes, planned comparisons were conducted on valid and invalid trials separately. On valid trials, participants responded as fast after goal-relevant cues as after all other cue categories, $t \mathrm{~s}(15)<1.52, n s$, suggesting that goal-relevant cues did not engage attention in comparison to the other categories. The differences between the other cue categories on valid trials were not significant, $t \mathrm{~s}(15)<1.01$. Only responses to goal-related cues on valid trials tended to be slower than responses to control-related cues on valid trials, $t(15)=1.82, p=$ $.089, d=0.56$

Analyses on invalid trials showed that participants responded significantly slower after goal-relevant cues than after all other cue categories, $t \mathrm{~s}(15)>2.46, p \mathrm{~s}<.03, d \mathrm{~s}>1.00$. This indicates delayed disengagement of attention from goal-relevant cues. The differences between the other cue categories on invalid trials were not significant, $t$ s $<1$.

\section{Discussion}

The present study examined whether goal-relevant words evoked larger cue validity effects than control words, even when attending to goal-relevant words during the cueing task was not functional and hence pursuit of the intention to attend to these words was not likely. Additionally, we examined the attentional processing of words that were semantically related to the goal-relevant words. In order to prevent that the semantic meaning of the goal-relevant words could be "turned off", the goal task required participants to react to a variety of goalrelevant pictures (i.e., pictures representing the content of the goal-relevant words) in addition to the goal-relevant words. The results replicated those of Vogt et al. (2010) in that goal-relevant but not goal-related cues caused larger cue validity effects compared to other control stimuli. These results therefore confirm the conclusion that goal-relevant but not goal-related stimuli attract attention in an unintended manner. 
The results of our study provide evidence for prominent accounts of automatic goal pursuit according to which goal-relevant stimuli attract attention automatically (Moskowitz et al., 2004; Ferguson et al., 2008). Our results suggest that attending to goal-relevant events is automatic in the sense of fast and unintended. Two arguments support the claim that participants did not hold an intention to attend more strongly to the goal-relevant words than to the other words in the spatial cueing task. First, goal-relevant words were task relevant in the goal task but not in the cueing task. Making the distinction between the two tasks salient, goal-relevant words in the goal task and goal-relevant cues in the cueing task were presented at clearly different locations and at clearly different moments in time. Second, unlike to what was the case in the study of Vogt et al. (2010), attending to (all) cues was not instrumental for the cueing task because cues did not predict the probe location above chance level ${ }^{3}$. If anything, heightened attending to the (goal-relevant) cues could only hamper performance during the cueing task. Hence, there is no reason to assume that participants had the intention to attend to the cues, let alone to attend specifically to the goal-relevant cues. By this, our results extend previous findings showing that attending to goal-relevant events is automatic in the sense of efficient (Moskowitz, 2002) or uncontrollable (Wieber \& Sassenberg, 2006).

Our results also convey which component of attentional orienting is affected by goalrelevant cues. First, goal-relevant words did not have a general distracting effect in that responses to probes were not significantly slower after goal-relevant cues than after the other cues. Second, the analyses of responses on invalid trials indicated that larger cue validity effects for goal-relevant words were due mainly to delayed disengagement of attention from goalrelevant words. Interestingly, other studies investigating attentional orienting to highly threatening (Fox et al., 2001) or highly arousing events (Vogt, De Houwer, Koster, Van Damme, \& Crombez, 2008) also revealed that such attentional biases are best characterized as a difficulty 
to disengage from this information. From a functional perspective, it makes sense that motivationally and goal-relevant stimuli evoke a difficulty to disengage attention because holding attention at a stimulus allows the organism to further process it and to react to it. Moreover, several authors suggest that attentional capture can only be found for events that are defined by simple visual features such as color (Stolz, 1996; Wolfe \& Horowitz, 2004).

Finally, our findings provide evidence for the selectivity of goal-driven but unintended attending. Previous studies hinted to the importance of shielding goal pursuit from interference by goal-irrelevant information. For instance, Shah and colleagues (2002) demonstrated that goal activation leads to the inhibition of information relevant to conflicting goals. Dreisbach and Haider (2008) demonstrated that, when goal-relevant events are well defined and are therefore well distinguishable from goal-irrelevant events, goal-irrelevant information will be neglected and does not interfere with goal-directed behavior. Furthermore, Veling and van Knippenberg (2006, 2008) found that the activation of a specific goal leads to the automatic inhibition of semantically related knowledge. Our study extends these findings by demonstrating that the early and automatic allocation of attention already differentiates between goal-relevant and goalrelated events. To the best of our knowledge, such a differentiation has never before been shown. Moreover, in contrast to Vogt et al. (2010), participants in the present experiment could not turn off the semantic meaning of the goal-relevant words (i.e., they had to remember what the words represented in order to identify the pictures and could not fulfill the goal task by identifying the visual features of the word). The present study therefore increases the ecological validity of this finding because in most real-life situations individuals are unlikely to forget the semantic meaning of goal-relevant events.

Interestingly, recent models of attention suggest that attention is automatically guided by highly activated representations in working and long-term memory to stimuli in the environment 
that match these representations (e.g., Desimone \& Duncan, 1995; Soto, Hodsoll, Rotshtein, \& Humphreys, 2008). Moreover, different findings (Stolz, 1996; Moores et al., 2003; Kiefer \& Martens, 2010) suggest that the activation of a semantic representation also leads to the preferred processing of information that is semantically related to this representation. Downing and Dodds (2004; see also Woodman \& Luck, 2007) extended this view by showing that guidance does not necessarily mean heightened attending but can also imply inattention. These authors found inattention to stimuli that matched activated information in working memory when this information was completely irrelevant for the currently performed task (i.e., information that had to be remembered for a subsequent task). Downing and Dodds (2004) reasoned that information that is activated but not currently relevant might get inhibited in order to prevent that attention is (mis)guided. Similarly, one could assume that also in our study representations of goal-relevant events were activated leading to heightened attention to goal-relevant events whereas the (lexical) representations of the synonyms were inhibited resulting in inattention to goal-related stimuli.

In conclusion, the present data provide further evidence that goal pursuit is accompanied by the operation of automatic processes. In particular, they show that goals influence already the first step of the processing of an incoming stimulus, that is, its attentional processing. Moreover, our findings show that this early level of attentional processing is selective, meaning that attention is allocated to goal-relevant events but not to goal-related events. 


\section{References}

Bargh, J. A. (1994). The four horsemen of automaticity: Awareness, intention, efficiency, and control in social cognition. In R. S. Wyer, Jr., \& T. K. Srull (Eds.), Handbook of social cognition (2nd ed.) (pp. 1-40). Hillsdale, NJ: Lawrence Erlbaum Associates, Inc.

Bargh, J. A. (1997). The automaticity of everyday life. In R. S. Wyer, Jr. (Ed.), Advances in social cognition (Vol. 10, pp. 1-61). Mahwah, NJ: Erlbaum.

Bargh, J. A., Gollwitzer, P. M., Lee-Chai, A., Barndollar, K., \& Troetschel, R. (2001). The automated will: Nonconscious activation and pursuit of behavioral goals. Journal of Personality and Social Psychology, 81, 1014-1027.

Chartrand, T. L. \& Bargh, J.A. (1996). Automaticity of impression formation and memorization goals. Journal of Personality and Social Psychology, 71, 464-478.

Cisler, J. M., \& Koster, E. H. W. (2010). Mechanisms of attentional biases towards threat in the anxiety disorders: An integrative review. Clinical Psychology Review, 30, 203216.

Corbetta, M., \& Shulman, G. L. (2002). Control of goal-directed and stimulus-driven attention in the brain. Nature Reviews Neuroscience, 3, 215-229.

Desimone, R., \& Duncan, J. (1995). Neural mechanisms of selective visual attention. Annual Review of Neuroscience, 18, 193-222.

Downing, P. E., \& Dodds, C. M. (2004). Competition in visual working memory for control of search. Visual Cognition, 11, 689-703.

Dreisbach, G., \& Haider, H. (2008). That's what task sets are for: Shielding against irrelevant information. Psychological Research, 72, 355-361.

Duncan, J., \& Humphreys, G. W. (1989). Visual search and stimulus similarity. Psychological Review, 96, 433-458. 
Duyck, W., Desmet, T., Verbeke, L., \& Brysbaert, M. (2004). WordGen: A tool for word selection and non-word generation in Dutch, German, English, and French. Behavior Research Methods, Instruments \& Computers, 36, 488-499.

Ferguson, M. J., Hassin, R., Bargh, J. A. (2008). Implicit motivation: Past, present, and future. In J. Y. Shah, \& W. L. Gardner (Eds.), Handbook of motivation science (pp. 150-165). New York, NY: Guilford.

Folk, C. L., Remington, R. W., \& Johnston, J. C. (1992). Involuntary covert orienting is contingent on attentional control settings. Journal of Experimental Psychology: Human Perception and Performance, 18, 1030-1044.

Förster, J., Liberman, N., \& Higgins, E. T. (2005). Accessibility from active and fulfilled goals. Journal of Experimental Social Psychology, 41, 220-239.

Fox, E., Russo, R., Bowles, R. J., \& Dutton, K. (2001). Do threatening stimuli draw or hold visual attention in sub-clinical anxiety? Journal of Experimental Psychology: General, $130,681-700$.

Hermans, D., \& De Houwer, J. (1994). Affective and subjective familiarity ratings of 740 Dutch words. Psychologica Belgica, 34, 115-140.

Inquisit 2.0 [Computer software]. (2005). Seattle, WA: Millisecond Software LLC.

Kiefer, M., \& Martens, U. (2010). Attentional sensitization of unconscious cognition: Task sets modulate subsequent masked semantic priming. Journal of Experimental Psychology: General, 139, 464-489.

Koster, E. H. W., Crombez, G., Van Damme, S., Verschuere, B., \& De Houwer, J. (2004). Does imminent threat capture and hold attention? Emotion, 4, 312-317.

Lang, P. J, Bradley, M. M., \& Cuthbert, B. (1999). International Affective Picture System (IAPS): Instruction Manual and Affective Ratings. University of Florida, NIMH Center 
for the Study of Emotion and Attention, Gainesville, FL, USA.

Luck, S. J. \& Vecera, S. P. (2002). Attention. In H. Pashler (Series Ed.) \& S. Yantis (Volume Ed.), Stevens' Handbook of Experimental Psychology: Vol. 1. Sensation and Perception (3rd ed., pp. 235-286). New York: Wiley.

Moores, E., Laita, L., \& Chelazzi, L. (2003). Associative knowledge controls deployment of visual selective attention. Nature Neuroscience, 6, 182-189.

Moors, A., \& De Houwer, J. (2001). Automatic appraisal of motivational valence: Motivational affective priming and Simon effects. Cognition and Emotion, 15, 749-766.

Moors, A., \& De Houwer, J. (2006). Automaticity: A theoretical and conceptual analysis. Psychological Bulletin, 132, 297-326.

Moskowitz, G. B. (2002). Preconscious effects of temporary goals on attention. Journal of Experimental Social Psychology, 38, 397-404.

Moskowitz, G. B., Li, P., \& Kirk, E. R. (2004). The implicit volition model: On the preconscious regulation of temporarily adopted goals. In M. P. Zanna (Ed.), Advances in experimental social psychology (pp. 317-414). San Diego, CA: Academic Press.

Ratcliff, R. (1993). Methods for dealing with reaction time outliers. Psychological Bulletin, 114, $510-532$.

Shah, J. Y. (2005). The automatic pursuit and management of goals. Current Directions in Psychological Science, 14, 10-13.

Shah, J. Y., Friedman, R., \& Kruglanski, A. W. (2002). Forgetting all else: On the antecedents and consequences of goal shielding. Journal of Personality and Social Psychology, 83, $1261-1280$.

Soto, D., Hodsoll, J., Rotshtein, P., \& Humphreys, G. W. (2008). Automatic guidance of attention from working memory. Trends in Cognitive Sciences, 12, 342-348. 
Stolz, J. A. (1996). Exogenous orienting does not reflect an encapsulated set of processes. Journal of Experimental Psychology: Human Perception \& Performance, 22, 187-201.

Veling, H., \& van Knippenberg, A. (2006). Shielding intentions from distraction: Forming an intention induces inhibition of distracting stimuli. Social Cognition, 24, 409-425.

Veling, H., \& van Knippenberg, A. (2008). Intention formation induces episodic inhibition of distracting stimuli. Acta Psychologica, 128, 45-55.

Vogt, J., De Houwer, J., Koster, E.H.W., Van Damme, S., \& Crombez, G. (2008). Allocation of spatial attention to emotional stimuli depends upon arousal and not valence. Emotion, 8 , $880-885$.

Vogt, J., De Houwer, J., Moors, A., Van Damme, S., \& Crombez, G. (2010). The automatic orienting of attention to goal-relevant stimuli. Acta Psychologica, 134, 61-69.

Vogt, J., De Houwer, J., \& Crombez, G. (in press). Multiple goal management starts with attention: Goal prioritising affects the allocation of spatial attention to goal-relevant events. Experimental Psychology. DOI 10.1027/1618-3169/a000066

Wieber, F., \& Sassenberg, K. (2006). I can’t take my eyes off of it - Attention attraction effects of implementation intentions. Social Cognition, 24, 723-752.

Wolfe, J. M., \& Horowitz, T. S. (2004). What attributes guide the deployment of visual attention and how do they do it? Nature Reviews Neuroscience, 5, 1-7.

Woodman, G. F. \& Luck, S. J. (2007). Do the contents of visual working memory automatically influence attentional selection during visual search? Journal of Experimental Psychology: Human Perception and Performance, 37, 363-377.

Yantis, S. (1998). Control of visual attention. In H. Pashler (Ed.), Attention (pp. 223-256). San Diego, CA: Psychology Press. 
Unintended attending to goal-relevant but not to goal-related events 22

\section{Author note}

Correspondence regarding this article should be addressed to Julia Vogt, Department of

Experimental-Clinical and Health Psychology, Ghent University, Henri Dunantlaan 2, 9000

Ghent, Belgium. E-mail: Julia.Vogt@UGent.be.

This research was funded by Grant BOF/GOA2006/001 of Ghent University. We would like to thank Anna Mester for her help in collecting the data. 


\section{Footnotes}

${ }^{1}$ Picture numbers were: $1610,1640,1660,2010,2270,2630,5870,5891,7002,7330,7351$, and 7450.

${ }^{2}$ The same effects were revealed when the medians of the RTs were used (cf. Ratcliff, 1993).

${ }^{3}$ The general cue validity effect was much weaker in this study $(M=13 \mathrm{~ms}, S D=23 \mathrm{~ms} ; F(1$, $\left.15)=5.07, p<.05, \mathrm{n}^{2}=.252\right)$ than in Experiment 2 of Vogt et al. $(M=33 \mathrm{~ms} ; S D=37 \mathrm{~ms} ; F(1$, $\left.29)=23.03, p<.001, \mathrm{y}^{2}=.443\right)$ in which $75 \%$ of the trials were valid. This suggests that participants in our study made little use of the cue position as a predictor for the probe position. 
Unintended attending to goal-relevant but not to goal-related events 24

Table 1

Mean Reaction Times, Standard Deviations, and Cue Validity Indices (in ms) as a Function of Cue Category and Cue Validity.

\begin{tabular}{lcccccc} 
& & \multicolumn{2}{c}{$\underline{\text { Valid }}$} & & \multicolumn{2}{c}{ Cue validity indices } \\
Cue category & $M$ & $S D$ & $M$ & $S D$ & $M$ & $S D$ \\
\hline Goal-relevant words & 416 & 60 & 447 & 70 & 31 & 30 \\
Goal-related words & 426 & 60 & 425 & 50 & -1 & 25 \\
Control words & 419 & 57 & 427 & 55 & 8 & 22 \\
Control-related words & 416 & 67 & 430 & 59 & 14 & 40
\end{tabular}

Note. Cue validity indices were calculated by subtracting reaction times on valid trials from reactions times on invalid trials. 


\section{Figure captions}

Figure 1. Schematic overview of two example trials of the combined spatial cueing and goal task. The first three boxes depict the spatial cueing task in which the presentation of a cue word was followed by a probe (black square) which had to be localized. The last two boxes display the goal task in which the presentation of a single word or picture was followed by the appearance of a question mark. Participants had to react to the question mark by pressing the spacebar when the single word or picture presented in the middle of the screen had been a goal-relevant word or picture. The left side illustrates a validly cued trial of the spatial cueing task, the right side an invalidly cued trial of this task. 
Figure 1.

TIME

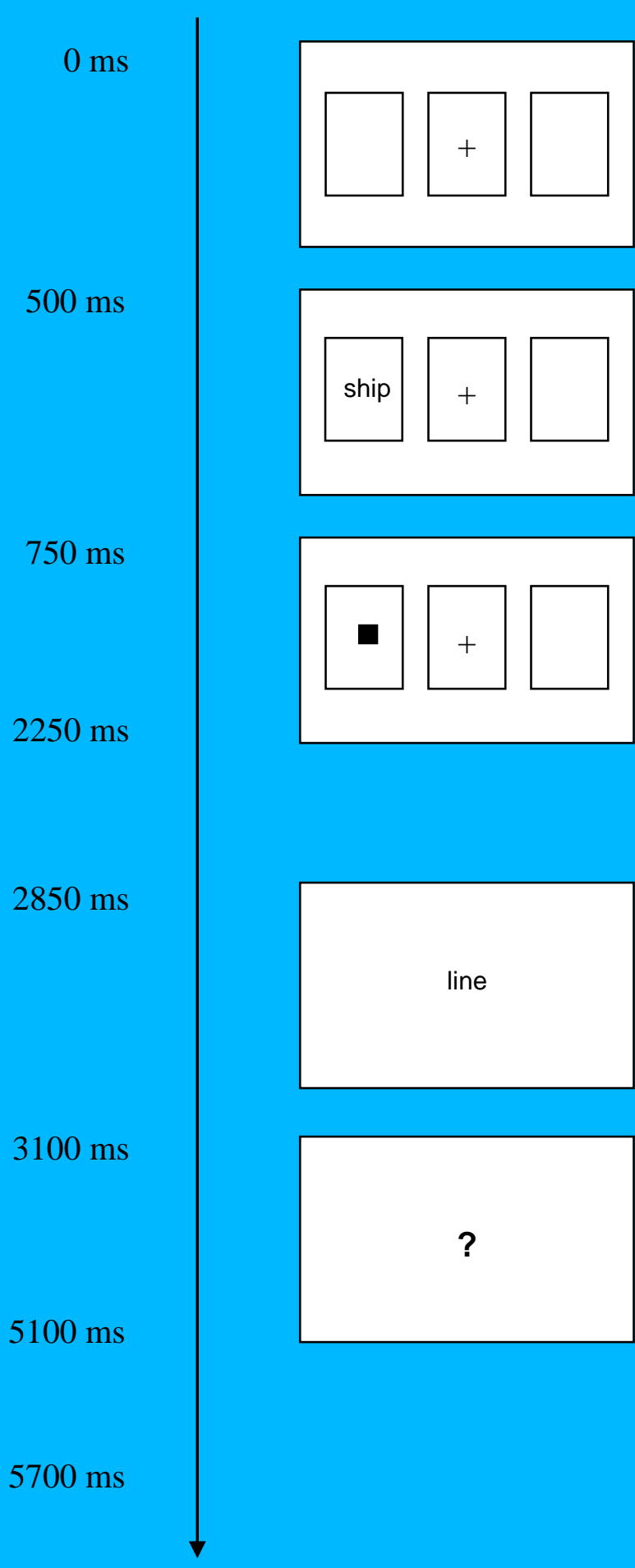

Invalidly cued
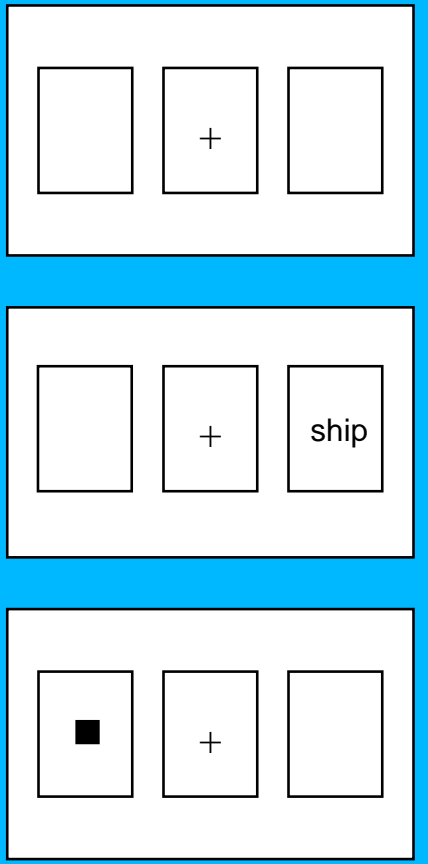

line

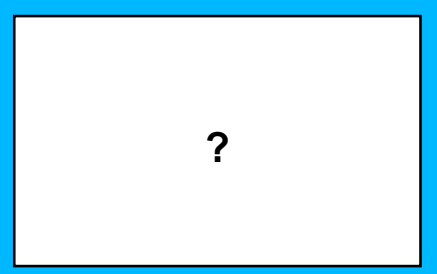

